\title{
Influence of the interplanetary magnetic field on the ring current injection rate
}

\author{
Takao Aoki \\ Nagano Technical High School, Sashide Minami 3-9-1, Nagano 380-0948, Japan \\ (Received February 7, 2005; Revised October 6, 2005; Accepted October 6, 2005; Online published May 12, 2006)
}

\begin{abstract}
In order to check the validity of Akasofu's $\varepsilon$ parameter and of the Vasyliunas et al. (1982) general formula, we examine the dependence of the ring current injection rate, calculated from the $D s t$ index for the period of 1965-1990, on the interplanetary magnetic field (IMF). We compare the influence of the $B z$ component with the influence of the combination of $\sin (\theta / 2)$, where $\theta$ is the IMF clock angle, and the IMF magnitude, $B$, (or the transverse component of the IMF, $\left.B_{T}=\left(B y^{2}+B z^{2}\right)^{1 / 2}\right)$ by using the regression analysis in a power law form. The main results are as follows: (1) the exponent for $B z$ shows higher consistency than that for $\sin (\theta / 2)$; (2) we never obtain $B^{2} \sin ^{4}(\theta / 2)$ or $B_{T}^{2} \sin ^{4}(\theta / 2)$, which is the IMF dependence expected from the $\varepsilon$ parameter; and (3) the ring current injection rate has a very low correlation with the Alfven Mach number, from which the IMF dependence of the Vasyliunas et al. general formula is assumed to arise. On the basis of the above results we conclude that the $\varepsilon$ parameter and the Vasyliunas et al. general formula are less appropriate than a function of $B z$, and that the energy coupling function between the solar wind and the Earth's magnetosphere is described better by $B z$ than by the combination of $B\left(\right.$ or $\left.B_{T}\right)$ and $\sin (\theta / 2)$. The above results and conclusions are the same as those obtained by Aoki (2005) through the analysis of the AL index.
\end{abstract}

Key words: Ring current injection rate, $\varepsilon$ parameter, Vasyliunas et al. general formula, IMF clock angle, $B z$.

\section{Introduction}

It is firmly established by a large number of researchers that the interplanetary magnetic field (IMF) plays a crucial role on the energy coupling between the solar wind and the Earth's magnetosphere. Fairfield and Cahill (1966) first showed that the $B z$ (north-south) component of the IMF generally controls the level of geomagnetic activity at high latitude observatories and that the southward direction is usually associated with disturbances. Perreault and Akasofu (1978) introduced the IMF clock angle $(\theta)$, which is defined as the angle that the projection of the IMF in the $Y-Z$ plane of the geocentric solar magnetospheric (GSM) coordinate system makes relative to the positive $Z$-axis, and emphasized its importance for the description of the energy coupling function because of the possibility of expressing the fact that the coupling can occur even for the northward IMF conditions. They used this angle to express their energy coupling function, the so-called epsilon parameter, $\varepsilon=l_{0}^{2} B^{2} V \sin ^{4}(\theta / 2)$, where $l_{0}$ is $7 R_{E}, B$ the IMF magnitude, $V$ the solar wind velocity, and $\varepsilon$ has the dimension of power. Since then this angle has been used as a fundamental parameter by a large number of researchers. Vasyliunas et al. (1982) performed dimensional analysis on the magnetohydrodynamic (MHD) flow, and obtained a general formula for the energy coupling function with the dimension of power. They derived their formula by assuming that the IMF dependence of the coupling function arises from the Alfven Mach number and the IMF clock angle. Various

Copyright (c) The Society of Geomagnetism and Earth, Planetary and Space Sciences (SGEPSS); The Seismological Society of Japan; The Volcanological Society of Japan; The Geodetic Society of Japan; The Japanese Society for Planetary Sciences; TERRAPUB. forms of coupling functions including the clock angle were proposed by a number of researchers through analyses of quantities, such as the AL index, the Dst index, and polar cap electric potentials (e.g., Gonzalez et al., 1994). The IMF dependence of these functions is usually expressed as the product of a power of $\sin (\theta / 2)$ and a power of the IMF magnitude, $B$, (or of the magnitude of the perpendicular component of the IMF, $\left.B_{T}=\left(B y^{2}+B z^{2}\right)^{1 / 2}\right)$.

On the other hand, some researchers proposed their coupling functions without using the clock angle. Examples of them are $B z V$ (Rostoker et al., 1972; Burton et al., 1975) and $B s V^{2}$ (Murayama and Hakamada, 1975; Maezawa and Murayama, 1986), where $B s$ is the southward component of the IMF, i.e., $B s=-B z$ for $B z<0$, and $B s=0$ for $B z \geq 0$. O'Brien and McPherron (2002) recently revised the Burton et al. equation to include the effect of the dipole tilt angle, but they did not use the clock angle. The above researchers express the IMF dependence of their coupling functions by the linear proportionality to $B z$.

However, comparison between the influence of $B z$ and the influence of the combination of $\sin (\theta / 2)$ and $B$ (or $B_{T}$ ) has not been made until recently. Aoki (2005) has made detailed comparisons by applying the regression analysis in a power law form to the AL index, and obtained the result that $B z$ shows superiority over the combination of $\sin (\theta / 2)$ and $B$ (or $\left.B_{T}\right)$. He has concluded that the IMF dependence of the AL index is described better by $B z$ than by the combination of $\sin (\theta / 2)$ and $B$ (or $B_{T}$ ), and that the $\varepsilon$ parameter and the Vasyliunas et al. general formula are less appropriate than a function of $B z$.

The purpose of the present paper is to make comparisons between the influence of $B z$ and that of the combination of 

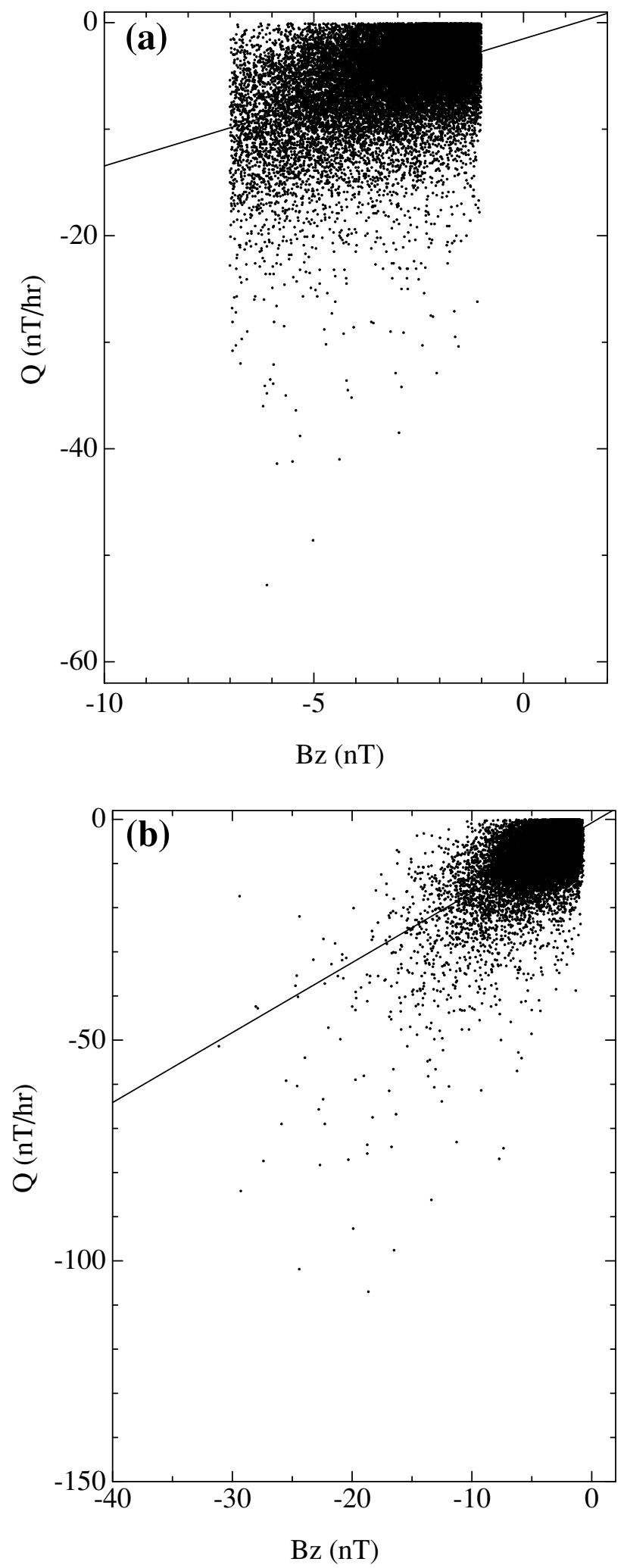

Fig. 1. (a) Scatter plot of $Q$ versus $B z$ for the data of $-7 \leq B z<-1$ nT and $V<600 \mathrm{~km} / \mathrm{s}$. The solid line is the regression line between $Q$ and $B z$. (b) Same as (a) but for the data of $B z<0 \mathrm{nT}$ and all $V$. (c) Same as (a) but for the data without the condition of (4) and without setting $Q=0$ even if the value of Eq. (3) is positive. Red points show the data for $E y \leq 0.50 \mathrm{mV} / \mathrm{m}$, and blue ones the data for $E y>0.50$ $\mathrm{mV} / \mathrm{m}$. (Purple points indicate the data in the region where both data coexist.) A red line is the regression line for the data of $E y \leq 0.50$ $\mathrm{mV} / \mathrm{m}, Q=0.038 B z-1.313$ with a correlation coefficient of 0.029 , and a blue one is for $E y>0.5 \mathrm{mV} / \mathrm{m}, Q=1.660 B z+0.119$ with a correlation coefficient of 0.625 .

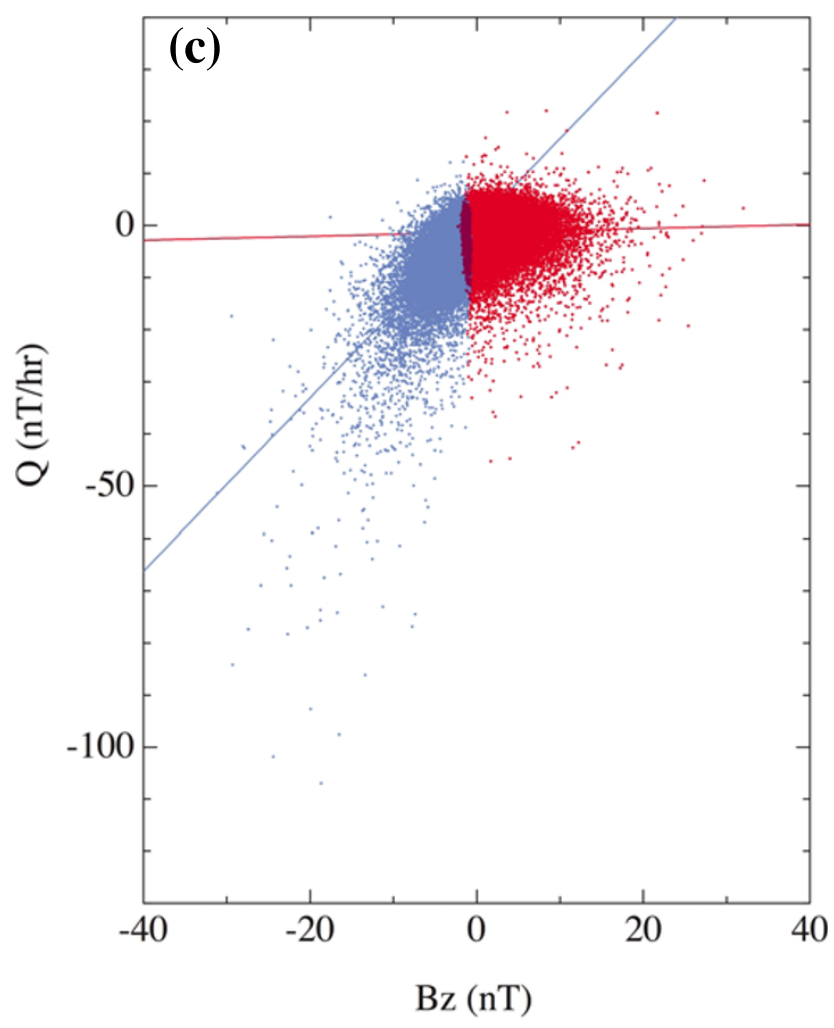

Fig. 1. (continued).

$\sin (\theta / 2)$ and $B_{T}$ (or $B$ ) by using the ring current injection rate calculated from the $D s t$ index, which is a measure of the magnitude of the ring current, and to examine whether or not the analysis of the injection rate leads to the same conclusion as that of the AL index (Aoki, 2005). We will show that the results of the ring current injection rate support the conclusion of the AL index.

\section{Data and Analysis}

\subsection{Data}

We use hourly values of the Dst index and of the solar wind parameters for the period of 1965-1990. We relate values of $D s t$ to solar wind parameters with thirty-minute delays by using averages of consecutive values of $D s t$. We introduce this time delay by taking account of the about 25 min response time of the Dst index to solar wind conditions (Burton et al., 1975).

According to Burton et al. (1975), the rate of injection into the ring current, $Q$, is related to the pressure-corrected $D s t, D s t^{*}$, by the following equation:

$$
Q=d D s t^{*} / d t+D s t^{*} / \tau \text {. }
$$

Here $D s t^{*}$ is given by

$$
D s t^{*}=D s t-b(P d)^{1 / 2}+c,
$$

where $P d$ is the solar wind dynamic pressure, $b$ a measure of the response to dynamic pressure changes in the solar wind, $c$ a measure of the quiet day currents, and $\tau$ the decay time of the ring current. The values of $b, c$, and $\tau$ are $0.20 \mathrm{nT}\left(\mathrm{eV} \cdot \mathrm{cm}^{-3}\right)^{-1 / 2}, 20 \mathrm{nT}$, and $2.78 \times 10^{4} \mathrm{~s}=7.7 \mathrm{hs}$, respectively. 
Table 1. Exponents and correlation coefficients (c.c.) for various kinds of solar wind parameters and of their combinations obtained by the regression analysis of the data for the range of $-7 \leq B z<-1 \mathrm{nT}$ and $V<600 \mathrm{~km} / \mathrm{s}$. In this table, $\left(B z^{\prime}, V, D\right)$, for example, represents the case of the regression equation of $\log (-Q)=$ const. $+\alpha_{1} \log \left(B z^{\prime}\right)+\alpha_{2} \log (V)+\alpha_{3} \log (D)$. Errors are the standard errors.

\begin{tabular}{|c|c|c|c|c|}
\hline \multirow{2}{*}{ Parameters } & \multicolumn{3}{|c|}{ Exponents } & \multirow{2}{*}{ c.c. ${ }^{*}$} \\
\hline & $\alpha_{1}$ & $\alpha_{2}$ & $\alpha_{3}$ & \\
\hline$B z^{\prime}$ & $1.049 \pm 0.018$ & & & 0.350 \\
\hline$B$ & $0.929 \pm 0.017$ & & & 0.340 \\
\hline$B_{T}$ & $0.599 \pm 0.013$ & & & 0.296 \\
\hline $\sin (\theta / 2)$ & $0.739 \pm 0.084$ & & & 0.058 \\
\hline$V$ & $1.287 \pm 0.035$ & & & 0.235 \\
\hline$M_{A}$ & $-0.165 \pm 0.012$ & & & -0.088 \\
\hline$M_{A}^{\prime}$ & $-0.152 \pm 0.016$ & & & -0.062 \\
\hline$\left(B z^{\prime}, V\right)$ & $1.182 \pm 0.018$ & $1.613 \pm 0.032$ & & 0.456 \\
\hline$(B, \sin (\theta / 2))$ & $1.123 \pm 0.018$ & $2.592 \pm 0.083$ & & 0.389 \\
\hline$\left(B_{T}, \sin (\theta / 2)\right)$ & $0.822 \pm 0.014$ & $3.116 \pm 0.088$ & & 0.367 \\
\hline$\left(M_{A}, \sin (\theta / 2)\right)$ & $-0.240 \pm 0.013$ & $1.366 \pm 0.091$ & & 0.132 \\
\hline$\left(M_{A}^{\prime}, \sin (\theta / 2)\right)$ & $-0.204 \pm 0.017$ & $1.018 \pm 0.087$ & & 0.098 \\
\hline$\left(B z^{\prime}, V, D\right)$ & $1.083 \pm 0.017$ & $2.161 \pm 0.034$ & $0.375 \pm 0.009$ & 0.512 \\
\hline$(B, \sin (\theta / 2), V)$ & $1.079 \pm 0.017$ & $2.685 \pm 0.081$ & $1.169 \pm 0.032$ & 0.444 \\
\hline$\left(B_{T}, \sin (\theta / 2), V\right)$ & $0.917 \pm 0.013$ & $3.620 \pm 0.084$ & $1.625 \pm 0.032$ & 0.470 \\
\hline$\left(M_{A}, \sin (\theta / 2), V\right)$ & $-0.358 \pm 0.013$ & $1.884 \pm 0.088$ & $1.514 \pm 0.035$ & 0.301 \\
\hline$\left(M_{A}^{\prime}, \sin (\theta / 2), V\right)$ & $-0.232 \pm 0.016$ & $1.243 \pm 0.085$ & $1.333 \pm 0.035$ & 0.262 \\
\hline
\end{tabular}

We approximate Eq. (1) by

$$
\begin{aligned}
Q(t)= & \left\{D s t^{*}(t+1 \text { hour })-D s t^{*}(t-1 \text { hour })\right\} / 2 \\
& +D s t^{*}(t) / 7.7 .
\end{aligned}
$$

We calculate this quantity for the hourly intervals in which all of the IMF, the solar wind velocity $(V)$, and the density $(D)$ are available. If values calculated from Eq. (3) are positive, we set $Q=0$. This is because the ring current is supposed to develop only for negative values of $Q$. We also impose the following restriction on the $y$ component of the solar wind electric field, $E y=-B z V$ (where $B z$ is measured in the GSM coordinates), according to Burton et al. (1975):

$$
Q=0 \quad \text { for } \quad E y \leq 0.50 \mathrm{mV} / \mathrm{m}
$$

This condition is considered as a cutoff below which the ring current does not develop. We use all negative values of $Q$ for the present analysis.

\subsection{Procedure of analysis}

Procedure of analysis in the present paper is basically the same as that of Aoki (2005) on the AL index.

Figures 1(a) and 1(b) are examples of scatter plots of $Q$ versus $B z$. Data of Fig. 1(a) and those of Fig. 1(b) are for $-7 \leq B z<-1 \mathrm{nT}$ and $V<600 \mathrm{~km} / \mathrm{s}$, and for $B z<0$ nT and all $V$, respectively. Data of Fig. 1(b) also correspond to the whole data of the present analysis. As is seen in these figures, $Q$ develops to large negative values as $B z$ becomes negative. The regression line of $Q$ on $B z$, however, usually does not pass through the origin of the $B z-Q$ plane, but tends to cross the $B z$-axis in the positive side of it. To take account of this tendency, we used the regression coefficients of the regression line, $Q=a B z+b$, to obtain the value, $B z_{0} \equiv-b / a$, as the value of the point at which the regression line crosses the abscissa. We use
$B z^{\prime}=B z_{0}-B z$ instead of $B z$ below. This rescaling from $B z$ to $B z^{\prime}$ is the same as that for the AL index (Aoki, 2005), and is considered as a way of describing the fact that the solar wind-magnetosphere coupling can occur even for the northward IMF conditions. The restriction on the data selection, $-7 \leq B z<-1 \mathrm{nT}$ and $V<600 \mathrm{~km} / \mathrm{s}$, was imposed to guarantee that the IMF is directed southward and thus the ring current injection is likely to occur, and to avoid extreme solar wind situations for statistically meaningful analyses.

For reference, Fig. 1(c) shows a scatter plot of $Q$ versus $B z$ for the data without the condition of (4) and without setting $Q=0$ even if the value of Eq. (3) is positive. Red points indicate the data for $E y \leq 0.50 \mathrm{mV} / \mathrm{m}$, and blue ones the data for $E y>0.50 \mathrm{mV} / \mathrm{m}$. If we remove the data of $E y \leq 0.50 \mathrm{mV} / \mathrm{m}$ and those of $Q \geq 0$, the remaining data become the same as those of Fig. 1(b). A red line gives the regression line for the data of $E y \leq 0.50 \mathrm{mV} / \mathrm{m}$, $Q=0.038 B z-1.313$, with a correlation coefficient of 0.029 . This very low correlation coefficient implies that there is almost no correlation between $Q$ and $B z$ for $E y \leq$ $0.5 \mathrm{mV} / \mathrm{m}$.

We performed the regression analysis in the form of $Y=a_{0} X_{1}^{\alpha_{1}}$ for each of the following parameters: $B z^{\prime}, B$, $B_{T}, \sin (\theta / 2), V, M_{A}$, and $M_{A}^{\prime}$, where $M_{A}$ and $M_{A}^{\prime}$ are the Alfven Mach numbers defined in terms of $B_{T}$ and of $B$, respectively, i.e., $M_{A}=D^{1 / 2} V / B_{T}$ and $M_{A}^{\prime}=D^{1 / 2} V / B$. This was done by the standard regression analysis using the equation $\log Y=\log a_{0}+\alpha_{1} \log X_{1}$. We also did the regression analysis in the form $Y=a_{0} X_{1}^{\alpha_{1}} X_{2}^{\alpha_{2}}$ for the following two-parameter combinations: $\left(B z^{\prime}, V\right),(B, \sin (\theta / 2))$, $\left(B_{T}, \sin (\theta / 2)\right),\left(M_{A}, \sin (\theta / 2)\right)$, and $\left(M_{A}^{\prime}, \sin (\theta / 2)\right)$. Furthermore we did the regression analysis in the form $Y=$ $a_{0} X_{1}^{\alpha_{1}} X_{2}^{\alpha_{2}} X_{3}^{\alpha_{3}}$ for the following three-parameter combinations: $\left(B z^{\prime}, V, D\right),(B, \sin (\theta / 2), V),\left(B_{T}, \sin (\theta / 2), V\right)$, 
Table 2. Exponents and correlation coefficients (c.c.) for $B z^{\prime}, \sin (\theta / 2),\left(B_{T}, \sin (\theta / 2)\right)$, and $\left(B z^{\prime}, V, D\right)$ for various ranges of $B z$ and for $V<600$ $\mathrm{km} / \mathrm{s}$.

\begin{tabular}{|c|c|c|c|c|c|c|c|c|c|c|c|c|c|}
\hline \multirow{2}{*}{$B z$ range } & \multirow{2}{*}{$\begin{array}{l}\text { Number of } \\
\text { data points }\end{array}$} & \multirow[b]{2}{*}{$B z_{0}$} & \multicolumn{2}{|l|}{$B z^{\prime}$} & \multicolumn{2}{|c|}{$\sin (\theta / 2)$} & \multicolumn{3}{|c|}{$\left(B_{T}, \sin (\theta / 2)\right)$} & \multicolumn{4}{|c|}{$\left(B z^{\prime}, V, D\right)$} \\
\hline & & & $\alpha$ & c.c. ${ }^{*}$ & $\alpha$ & c.c. ${ }^{*}$ & $\alpha_{1}$ & $\alpha_{2}$ & c.c. ${ }^{*}$ & $\alpha_{1}$ & $\alpha_{2}$ & $\alpha_{3}$ & c.c. ${ }^{*}$ \\
\hline$[-6, \quad 0]$ & 22135 & 1.6 & $1.04 \pm 0.02$ & 0.304 & $0.40 \pm 0.09$ & 0.032 & $0.76 \pm 0.02$ & $2.78 \pm 0.09$ & 0.323 & $1.12 \pm 0.02$ & $2.17 \pm 0.04$ & $0.38 \pm 0.01$ & 0.485 \\
\hline$\left[\begin{array}{ll}-7, & 0\end{array}\right]$ & 23188 & 1.3 & $1.03 \pm 0.02$ & 0.347 & $0.74 \pm 0.08$ & 0.058 & $0.80 \pm 0.01$ & $3.03 \pm 0.09$ & 0.363 & $1.08 \pm 0.02$ & $2.17 \pm 0.03$ & $0.38 \pm 0.01$ & 0.511 \\
\hline$\left[\begin{array}{ll}, & 0\end{array}\right]$ & 25121 & 0.4 & $0.91 \pm 0.01$ & 0.461 & $1.72 \pm 0.08$ & 0.129 & $0.91 \pm 0.01$ & $3.55 \pm 0.08$ & 0.476 & $0.89 \pm 0.01$ & $2.14 \pm 0.03$ & $0.37 \pm 0.01$ & 0.586 \\
\hline$[-6,-1]$ & 21965 & 1.6 & $1.07 \pm 0.02$ & 0.307 & $0.40 \pm 0.09$ & 0.032 & $0.78 \pm 0.02$ & $2.88 \pm 0.09$ & 0.327 & $1.13 \pm 0.02$ & $2.16 \pm 0.04$ & $0.38 \pm 0.01$ & 0.485 \\
\hline$[-7,-1]$ & 23018 & 1.3 & $1.05 \pm 0.02$ & 0.350 & $0.74 \pm 0.08$ & 0.058 & $0.82 \pm 0.01$ & $3.12 \pm 0.09$ & 0.367 & $1.08 \pm 0.02$ & $2.16 \pm 0.03$ & $0.38 \pm 0.01$ & 0.512 \\
\hline$[\quad,-1]$ & 24951 & 0.4 & $0.93 \pm 0.01$ & 0.465 & $1.73 \pm 0.08$ & 0.129 & $0.93 \pm 0.01$ & $3.62 \pm 0.08$ & 0.480 & $0.90 \pm 0.01$ & $2.13 \pm 0.03$ & $0.37 \pm 0.01$ & 0.587 \\
\hline$[-6,-2]$ & 15271 & 0.7 & $1.13 \pm 0.03$ & 0.306 & $-0.08 \pm 0.12$ & -0.005 & $1.05 \pm 0.02$ & $4.04 \pm 0.14$ & 0.330 & $1.06 \pm 0.03$ & $2.10 \pm 0.04$ & $0.37 \pm 0.01$ & 0.490 \\
\hline$[-7,-2]$ & 16324 & 0.5 & $1.11 \pm 0.02$ & 0.354 & $0.29 \pm 0.11$ & 0.020 & $1.09 \pm 0.02$ & $4.21 \pm 0.13$ & 0.374 & $1.04 \pm 0.02$ & $2.09 \pm 0.04$ & $0.37 \pm 0.01$ & 0.519 \\
\hline$[\quad,-2]$ & 18257 & -0.2 & $1.00 \pm 0.01$ & 0.488 & $1.48 \pm 0.11$ & 0.097 & $1.14 \pm 0.02$ & $4.51 \pm 0.11$ & 0.503 & $0.90 \pm 0.01$ & $2.01 \pm 0.04$ & $0.35 \pm 0.01$ & 0.603 \\
\hline
\end{tabular}

$\left(M_{A}, \sin (\theta / 2), V\right)$, and $\left(M_{A}^{\prime}, \sin (\theta / 2), V\right)$.

We performed the above analyses under various conditions on $B z$ and $V$. As the conditions on $B z$, we chose the following nine ranges: $[-6,0]$ (which means $-6 \leq$ $B z<0 \mathrm{nT}$; the same format is used throughout this paper), [-7,0], [ ,0] (which means $B z<0$ nT), [-6, -1], $[-7,-1],[,-1],[-6,-2],[-7,-2]$, and $[,-2]$. As the conditions on $V$, we examined the following three ranges: $V<600 \mathrm{~km} / \mathrm{s}, V \geq 600 \mathrm{~km} / \mathrm{s}$, and all $V$. We analyzed all cases of any combinations of the above $B z$ and $V$ ranges. We will compare the exponents and the correlation coefficients in the next section.

\section{Results}

Table 1 shows the exponents and the correlation coefficients for various solar wind parameters and their combinations for the range of $-7 \leq B z<-1 \mathrm{nT}$ and $V<$ $600 \mathrm{~km} / \mathrm{s}$. This range of $B z$ and $V$ is chosen as a typical example. From this table, we notice the following points:

1) The combination $\left(B z^{\prime}, V, D\right)$ shows the highest correlation coefficient among the quantities listed in Table 1 .

2) The correlation coefficients for $M_{A}, M_{A}^{\prime}$, $\left(M_{A}, \sin (\theta / 2)\right)$, and $\left(M_{A}^{\prime}, \sin (\theta / 2)\right)$ are very low compared with that for $B z^{\prime}$, and the correlation coefficients for $\left(M_{A}, \sin (\theta / 2), V\right)$ and $\left(M_{A}^{\prime}, \sin (\theta / 2), V\right)$ are clearly low compared with that for $\left(B z^{\prime}, V\right)$ and even compared with that for $B z^{\prime}$.

3) The exponent for $B z^{\prime}$ is about unity in all cases including $B z^{\prime}$, i.e., $B z^{\prime},\left(B z^{\prime}, V\right)$, and $\left(B z^{\prime}, V, D\right)$.

4) The exponent for $\sin (\theta / 2)$ varies over a wide range for the change in the combination of parameters.

5) The exponent for $\mathrm{V}$ varies from about unity to two.

The above features were confirmed to be true for other ranges of $B z$ and $V$.

Table 2 shows the examples of the analysis for various kinds of solar wind parameters and their combinations: the exponents and the correlation coefficients for various ranges of $B z$ and for $V<600 \mathrm{~km} / \mathrm{s}$. From this table, we notice the following facts:
1) The exponent for $B z^{\prime}$ is about unity. This character remains valid for the change in the range of $B z$.

2) The exponent for $\sin (\theta / 2)$ shows large variability for the change in the range of $B z$.

3) Concerning the combination $\left(B z^{\prime}, V, D\right)$, the exponents for $B z^{\prime}, V$, and $D$ show almost no variability for the change in the range of $B z$; the exponents for $B z^{\prime}$ are about unity, those for $V$ are about two, and those for $D$ are about 0.38 . It is worth pointing out that these values are very close to the values obtained by Maezawa and Murayama (1986), who showed that the best exponents for $B s$ (the southward component of the IMF), $V$, and $D$ are $1.09,2.06$, and 0.38 , respectively, through the analysis of selected storm events.

We would like to point out one fact. From the present analysis described in the preceding section, we never obtained $B_{T}^{2} \sin ^{4}(\theta / 2)$ or $B^{2} \sin ^{4}(\theta / 2)$, which is the IMF dependence expected from the $\varepsilon$ parameter. This result is the same as that obtained by Aoki (2005) on the AL index. Thus we can conclude that the IMF dependence of the $\varepsilon$ parameter is never established empirically by the analyses of AL or of $Q$.

\section{Discussion}

4.1 On the validity of the $\varepsilon$ parameter and of the Vasyliunas et al. general formula

In this subsection we discuss the validity of the $\varepsilon$ parameter and of the Vasyliunas et al. (1982) general formula on the basis of the results of the preceding section.

First, we consider the $\varepsilon$ parameter. As mentioned in the last part of the preceding section, we never obtained $B^{2} \sin ^{4}(\theta / 2)$ or $B_{T}^{2} \sin ^{4}(\theta / 2)$ by the logarithmic analysis with restrictions on the values of $B z$. Here, we check this result by the linear analysis with restrictions on the values of $\theta$, not on the values of $B z$. We imposed restrictions on $\theta$ by $90-10 p \leq \theta<90+10 p^{\circ}(p=1,2, \cdots, 9)$, and for each range of $\theta$ we performed the linear regression analysis of $Q$ on $V^{l} B^{m} \sin ^{n}(\theta / 2)(l=0,1 ; m=0,2 ; n=1,2,4)$, on $V^{l} B_{T}^{m} \sin ^{n}(\theta / 2)(l=0,1 ; m=0,2 ; n=1,2,4)$, and 
(a)

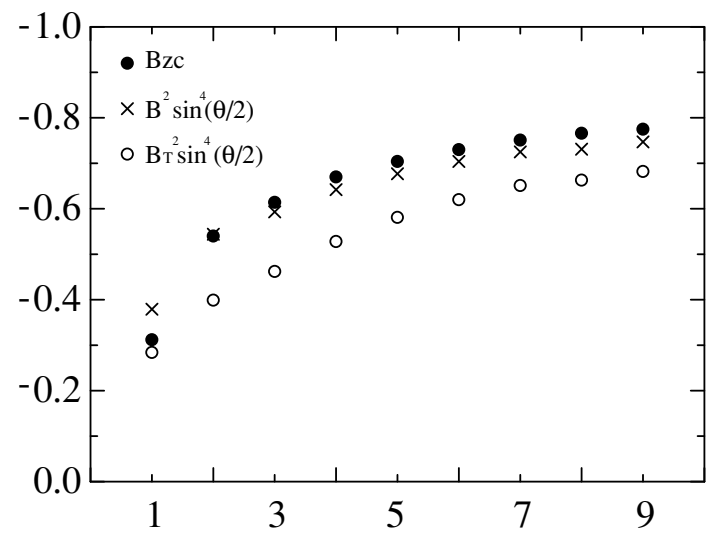

(b)

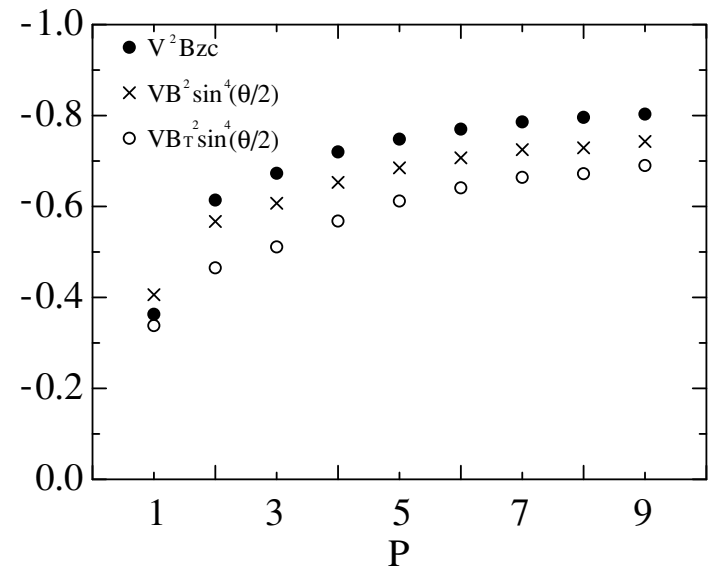

Fig. 2. (a) Correlation coefficients (c.c.) of $\mathrm{Q}$ with $B z c$ (solid circles), with $B^{2} \sin ^{4}(\theta / 2)$ (crosses), and with $B_{T}^{2} \sin ^{4}(\theta / 2)$ (open circles) for the ranges of $90-10 p \leq \theta \leq 90+10 p^{\circ},(p=1,2, \cdots, 9)$ and for the range of $V<600 \mathrm{~km} / \mathrm{s}$. Values of the abscissa are the values of $p$. (b) Correlation coefficients (c.c.) of $Q$ with $V^{2} B z c$ (solid circles), with $V B^{2} \sin ^{4}(\theta / 2)$ (crosses), and with $V B_{T}^{2} \sin ^{4}(\theta / 2)$ (open circles) for the ranges of $90-10 p \leq \theta \leq 90+10 p^{\circ},(p=1,2, \cdots, 9)$ and for the range of $V<600 \mathrm{~km} / \mathrm{s}$.

on $V^{l} B z c(l=0,2)$, where $B z c$ is defined by

$$
\begin{array}{lll}
B z c=0.8-B z & \text { for } & B z<0.8 \mathrm{nT} \\
B z c=0 & \text { for } & B z \geq 0.8 \mathrm{nT}
\end{array}
$$

Here, 0.8 is the average of the values of $B z_{0}$ in Table 2. We did this analysis for the following three ranges of $V: V<$ $600 \mathrm{~km} / \mathrm{s}, V \geq 600 \mathrm{~km} / \mathrm{s}$, and all $V$. Figures 2(a) and 2(b) show the comparison among $B^{2} \sin ^{4}(\theta / 2), B_{T}^{2} \sin ^{4}(\theta / 2)$, and $B z c$, and that among $V B^{2} \sin ^{4}(\theta / 2), V B_{T}^{2} \sin ^{4}(\theta / 2)$, and $B z c V^{2}$, respectively, for the range of $V<600$ $\mathrm{km} / \mathrm{s}$. From these figures, general superiority of $B z$ over $B^{2} \sin ^{4}(\theta / 2)$ and over $B_{T}^{2} \sin ^{4}(\theta / 2)$ is evident. Other results (not shown) also support the conclusion that $B z$ is better than the combination of $B$ (or $\left.B_{T}\right)$ and $\sin (\theta / 2)$.

Next, we consider the general formula of Vasyliunas et al. (1982). This formula is derived on the assumption that the IMF dependence of the coupling function arises from the Alfven Mach number and $\sin (\theta / 2)$. However, as was pointed out in the preceding section, $M_{A}, M_{A}^{\prime},\left(M_{A}, \sin (\theta / 2)\right)$, and $\left(M_{A}^{\prime}, \sin (\theta / 2)\right)$ have much smaller correlation coefficients with $Q$ than $B z^{\prime}$, and $\left(M_{A}, \sin (\theta / 2), V\right)$ and $\left(M_{A}^{\prime}, \sin (\theta / 2), V\right)$ also have clearly smaller correlation coefficients than $\left(B z^{\prime}, V\right)$. These facts
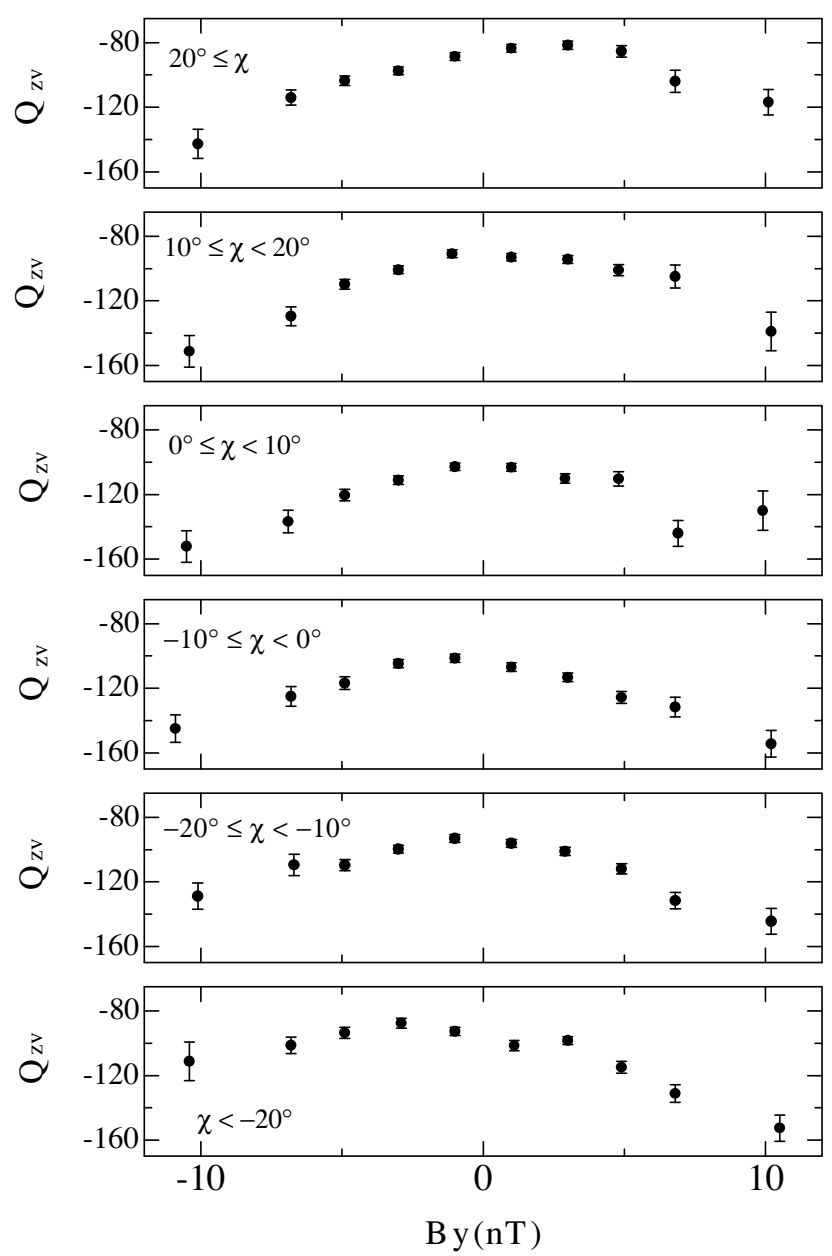

Fig. 3. Dependence of $Q_{Z V} \equiv\left(Q /\left(B z^{\prime} V^{* 1.6}\right)\right) \times 100$, where $V^{*} \equiv V / 400$, on $B y$ for different ranges of $\chi$ for the data of $-7 \leq B z<-1 \mathrm{nT}$ and $V<600 \mathrm{~km} / \mathrm{s}$. Error bars indicate the standard errors.

imply that the Alfven Mach number does not play any important role in the ring current injection and hence in the energy coupling between the solar wind and the Earth's magnetosphere. (See Appendix for more detailed discussion.)

Lastly, we discuss the idea that the IMF dependence of the coupling function can be described by the combination of $\sin (\theta / 2)$ and $B$ (or $B_{T}$ ). Aoki (2005) pointed out that this idea has a difficulty in expressing the combined effect of the IMF $B y$ component and the dipole tilt angle $(\chi)$ on the AL index. The reason is as follows: AL develops more efficiently for positive $B y$ than for negative $B y$ when $\chi$ is negative, and vice versa when $\chi$ is positive (Aoki, 1977; Murayama et al., 1980). Below we refer to this effect as the $B y-\chi$ effect. This effect is obviously asymmetric with respect to the sign of $B y$. Each of the quantities of $B, B_{T}$, and $\sin (\theta / 2)$, however, is symmetric with respect to it, so it is impossible to describe the $B_{y}-\chi$ effect by the combination of $\sin (\theta / 2)$ and $B$ (or $\left.B_{T}\right)$.

Here, we would like to check whether or not $Q$ has the $B y-\chi$ effect. Figure 3 shows the $B y$ dependence of $Q z v \equiv$ $Q /\left(B z^{\prime} V^{* 1.6}\right) \times 100, Q$ corrected for the effect of $B z$ and of $V^{*}(\equiv V / 400)$, for the data of $-7 \leq B z<-1 \mathrm{nT}$ and $V<600 \mathrm{~km} / \mathrm{s}$ separately for different ranges of $\chi$. From this figure, we notice that $Q z v$ tends to develop as the 
Table 3. Linear correlation coefficients (c.c.) between solar wind parameters for various ranges of $B z$ and for $V<600 \mathrm{~km} / \mathrm{s}$. In this table, $\langle D, V\rangle$, for example, represents the case for the linear regression analysis between $D$ and $V$. The same data as those in Table 2 are used for each range of $B z$.

\begin{tabular}{|c|c|c|c|c|c|c|}
\hline$B z$ range & $\begin{array}{l}\text { Number of } \\
\text { data points }\end{array}$ & $\langle D, B z\rangle$ & $\langle D, B\rangle$ & $\left\langle D, B_{T}\right\rangle$ & $\langle D, \sin (\theta / 2)\rangle$ & $\langle D, V\rangle$ \\
\hline$[-6, \quad 0]$ & 22135 & -0.155 & 0.249 & 0.218 & -0.037 & -0.353 \\
\hline$[-7,0]$ & 23188 & -0.171 & 0.262 & 0.229 & -0.026 & -0.349 \\
\hline$\left[\begin{array}{ll}\quad, & 0\end{array}\right]$ & 25121 & -0.250 & 0.316 & 0.283 & 0.017 & -0.325 \\
\hline$[-6,-1]$ & 21965 & -0.149 & 0.248 & 0.215 & -0.042 & -0.351 \\
\hline$[-7,-1]$ & 23018 & -0.167 & 0.261 & 0.227 & -0.031 & -0.346 \\
\hline$[\quad,-1]$ & 24951 & -0.246 & 0.314 & 0.281 & 0.013 & -0.321 \\
\hline$[-6,-2]$ & 15271 & -0.086 & 0.229 & 0.185 & -0.099 & -0.331 \\
\hline$[-7,-2]$ & 16324 & -0.110 & 0.242 & 0.197 & -0.088 & -0.326 \\
\hline$[\quad,-2]$ & 18257 & -0.215 & 0.300 & 0.257 & -0.039 & -0.293 \\
\hline
\end{tabular}

absolute values of $B y$ increase in every range of $\chi$, and that it also tends to develop more efficiently for positive $B y$ than for negative $B y$ when $\chi$ is negative, and vice versa when $\chi$ is positive. Thus $Q$ has the $B y-\chi$ effect.

The cause of the $B y-\chi$ effect is an open question. One possibility is as follows: As pointed out by Murayama et al. (1980) and by Nakai (1987), we may expect that the location of reconnection at the dayside magnetopause shifts to the pre-noon or post-noon side depending on the sign of the $B y$ component of the IMF, and also moves to the summer hemisphere side in association with the variation of $\chi$. According to the $B y-\chi$ effects of AL and of $Q$, the period in which $\mathrm{AL}$ and $Q$ develop more efficiently is the time when reconnection occurs on the dawn side of the magnetopause. So we can understand the $B y-\chi$ effects of $\mathrm{AL}$ and of $Q$ by assuming that reconnection on the dawn side of the magnetopause works more efficiently than that on the dusk side for some reason.

From the above considerations, we conclude that the $\varepsilon$ parameter and the Vasyliunas et al. (1982) general formula are less appropriate than a function of $B z$, and that the energy coupling function between the solar wind and the Earth's magnetosphere is described better by $B z$ than by the combination of $B$ (or $B_{T}$ ) and $\sin (\theta / 2)$. It is worth mentioning that the above results and conclusions are the same as those obtained by Aoki (2005) through the analysis of the AL index. On the basis of the above results, with attention to the fact that the effect of $B y$ is small compared with that of $B z$ (cf. Figs. 1 and 3), we suggest that the coupling function, $P$, is approximated by

$$
P=f(B y, \chi) B z^{\prime \alpha} V^{\beta} D^{\gamma},
$$

where $\alpha \sim 1, \beta \sim 2, \gamma \sim 0.4$, and $f(B y, \chi)$ is a function for expressing the effects of $B y$ and of $\chi$.

\subsection{Influence of the intercorrelations among solar} wind parameters

In this subsection we discuss the influence of the intercorrelations among solar wind parameters on the results.

The intercorrelations among $B z, B, B_{T}, \sin (\theta / 2)$, and $V$ were examined by Aoki (2005), and they are not very different from the intercorrelations for the data of the present analysis (not shown). Aoki (2005) did not discuss intercorrelations including the solar wind density because the dependence of the AL index on the density is weak. The solar wind density, however, has a stronger influence on $Q$ (cf. Table 1) than on $\mathrm{AL}$, so we examine its intercorrelations here. Table 3 shows the linear correlation coefficients for the combinations of $(D, B z),(D, B),\left(D, B_{T}\right)$, $(D, \sin (\theta / 2))$, and $(D, V)$ for different ranges of $B z$ and for $V<600 \mathrm{~km} / \mathrm{s}$. As is seen in this table, the solar wind density has weak correlations with $B z, B, B_{T}$, and $V$. Among them the intercorrelation between $D$ and $V$ is relatively high (about an anticorrelation between $V$ and $D$, see, e.g., Neugebaur and Snyder, 1966).

The influence of the intercorrelation between $D$ and $V$ and of the strong $D$ dependence of $Q$ can be seen in a rather large difference in the exponent for $V$ between $\left(B z^{\prime}, V\right)$ and $\left(B z^{\prime}, V, D\right)$. The exponent for $V$ for the case of $\left(B z^{\prime}, V, D\right), 2.16$, is larger than that for the case of $\left(B z^{\prime}, V\right), 1.61$ (cf. Table 1$)$; the difference is 0.55 . Thus to get the accurate exponent for $V$ we should include the influence of the solar wind density. This value of about two of the exponent for $V$ supports the quadratic dependence on $V$ (Murayama and Hakamada, 1975; Maezawa and Murayama, 1986), but does not support the linear dependence on $V$, which is expected from the $\varepsilon$ parameter.

\subsection{Influence of the assumptions for calculating the ring current injection rate}

We have analyzed $Q$ derived with some assumptions described in Section 2.1. In this subsection we discuss the influence of some of those assumptions on the results.

First, we consider the influence of the condition on $E y$, Eq. (4). In order to evaluate this influence, we calculated a new injection rate without this condition, and performed the same analysis as that in the above. Elimination of the condition of Eq. (4) yielded increase in the number of data points in the small negative $B z$ ranges, but almost no change in the number of data points in the large negative $B z$ ranges. Through the analysis of this injection rate, we have confirmed that all of the features listed in Section 3 are generally valid, although there are some small changes in exponents and in correlation coefficients. We also have confirmed the existence of the $B y-\chi$ effect. 
Second, a number of researchers suggested that the decay time is not a constant but has dependence on a parameter: its dependence on the value of Dst (Feldstein et al., 1984; Gonzalez et al., 1989), and on Ey (Fenrich and Luhmann, 1998; O'Brien and McPherron, 2000). We discuss these possibilities here. We investigated the following three decay times suggested by Gonzalez et al. (1989), by Fenrich and Luhmann (1998), and by O'Brien and McPherron (2000):

$$
\begin{array}{rlrl}
\tau_{G} & =4 \mathrm{hs} & & \text { for } \quad-50 \mathrm{nT} \leq D s t \\
& =0.5 \mathrm{hs} & & \text { for } \quad-120 \leq D s t<-50 \mathrm{nT} \\
& =0.25 \mathrm{hs} & & \text { for } \quad D s t<-120 \mathrm{nT}, \\
\tau_{F L} & =4 \mathrm{hs} & & \text { for } \quad E y>4 \mathrm{mV} / \mathrm{m} \\
& =7.7 \mathrm{hs} & & \text { for } \quad E y \leq 4 \mathrm{mV} / \mathrm{m}, \\
\tau_{O M} & =2.4 \exp \{9.7 /(4.7+E y)\} \mathrm{hs} .
\end{array}
$$

We have performed the same analysis as that in the above, and confirmed the validity of all of the features listed in Section 3 and the existence of the $B y-\chi$ effect for each of the injection rates derived by using $\tau_{G}, \tau_{F L}$, and $\tau_{O M}$, except for the fact that the exponent for $V$ for the injection rate of $\tau_{G}$ varies between two and four, being larger than those for the injection rates of other $\tau$ 's.

Here, we would like to point out two facts concerning $\tau_{G}$ : (1) the value of $\tau_{G}$ is rather small compared with those of other $\tau$ 's, and (2) the injection rate of $\tau_{G}$ has a clearly lower linear correlation coefficient with $B z$ than those of other $\tau$ 's and than that of $\tau=7.7 \mathrm{hs}$. Detailed comparison is beyond the scope of the present paper, and should be discussed in a separate paper.

\subsection{On the method of analysis of the present study}

4.4.1 On the method of the regression analysis in a logarithmic form In the present study we have examined the dependence of $Q$ on the solar wind parameters, mainly on the IMF, by using the regression analysis in a logarithmic form. In this subsection we discuss the characters of this method and compare them with those of previous studies.

Regression analysis in a logarithmic form has a feature that it can generally deal with every exponent (which is equal to the regression coefficient in this analysis) of parameters of interest, and that it can give the most probable value of each exponent. Coupling functions proposed so far are usually expressed as the products of powers of some solar wind parameters, and there are some controversies about values of exponents (e.g., $V$ versus $V^{2}$ ). Our method easily judges the appropriateness of those values of exponents.

Wu and Lundsted (1997a, b) investigated the influence of the solar wind parameters on the Dst index by using the neural network method. They showed that the two basic combinations giving accurate prediction are $(B z, V, D)$ and $(B s, V, D)$, and that $\varepsilon$ is less appropriate. These results are consistent with ours. In the neural network method, however, an exponent of each parameter should be given before performing the analysis, and so it is not very easy to say what is the most appropriate value of the exponent for the solar wind parameter of interest.

We have investigated the IMF dependence of $Q$ without considering detailed dependence of $Q$ on $V$ and on $D$. This treatment is guaranteed by the fact that the IMF-related parameters (i.e., $B z, B, B_{T}$, and $\left.\sin (\theta / 2)\right)$ have almost no correlations with $V$ (Aoki, 2005). This treatment is different from that of studies in which an exact form of the coupling function including all of the parameters which are assumed to have influence is given first and the validity of this function is examined. Examples of those studies are O'Brien and McPherron (2000) and Temerin and Li (2002). Their purpose is to find out a suitable function that describes the whole time evolution (i.e., the development and the decay) of $D s t$, but our objective is to seek suitable parameters for describing the IMF dependence of the coupling function when the injection occurs, i.e., when $Q<0$.

Lastly, we discuss the requirement that the coupling function have the dimension of power (Perreault and Akasofu, 1978; Vasyliunas et al., 1982). It is not necessarily clear that the result of the regression analysis in a logarithmic form meets this requirement. Here, it is worth noting the following two points: First, $Q$ physically reflects the total kinetic energy of the ring current particles through the Dessler-Parker-Sckopke relation (Dessler and Parker, 1959; Sckopke, 1966). However, $Q$ does not have the dimension of power because of its definition of Eq. (1). Second, what the dimension of $Q$ is is a separate problem from what are parameters controlling $Q$. If the intensification of the ring current is a result of the solar wind-magnetosphere coupling, $Q$ should show dependence on the same parameters as those controlling the coupling. Vasyliunas et al. (1982) assumed that the coupling depends on the Alfven Mach number and on the clock angle. If this assumption is correct, $Q$ should also depend on those parameters irrespective of the dimension of $Q$. (A similar argument can also be applied to the AL index.) The present study addresses the problem of the validity of this assumption, and the regression analysis can give a clear answer to this problem.

4.4.2 Time resolution of the data In the present study we have used hourly values of $Q$, and often compared the results of $Q$ with those of hourly values of AL (Aoki, 2005). In this subsection we discuss the influence of the time resolution of the data on the result and the physical meaning of the comparison between the results of $Q$ and those of AL.

Studies of the coupling function by using the AE, AL, and $D s t$ indices have a long history. Typical analyses of AE or of AL were performed by using 1-min values (e.g., Baker et al., 1983), and 3-h values of the indices (e.g., Maezawa, 1979). Irrespective of the time resolutions of the data, these studies gave similar dependence on solar wind parameters, at least on the IMF and on $V$. Typical analyses on $D s t$ were done by using 2.5-min values (e.g., Burton et al., 1975) and 1-h values of the index (e.g., Gonzaletz et al., 1989). These studies on Dst led to similar dependence on the IMF and on $V$ to each other. Furthermore, Maezawa and Murayama (1986) showed that the analysis of 3-h values of AL and that of 1-h values of Dst yielded similar dependence on the IMF and on $V$. These similarities suggest that analyses of AL and of Dst lead to almost the same coupling function as long as the time resolution of the indices is between 1 minute and about three hours and that the two indices reflect consequences of the same mechanism of the solar windmagnetosphere coupling. However, an analysis similar to the present study with high time resolution data has never 
been performed before, so it should be done in a future study to check the above idea.

\subsection{Previous studies on the Vasyliunas et al. formula and the $\varepsilon$ parameter}

In this subsection we discuss some of previous investigations that dealt with the Vasyliunas et al. general formula and $\varepsilon$.

Murayama (1986) analytically derived an equation for $Q$ in the form of $B s^{\alpha} V^{\beta} D^{\gamma}$, and compared its exponents with those expected from the Vasyliunas et al. formula. From this comparison he concluded that the values of exponents of his equation are inconsistent with those of the formula. He further suggested that this inconsistency is avoided by introducing an additional multiplicative factor depending on $B s V$. This procedure, however, is inappropriate because of the following reason: If the Vasyliunas et al. formula is truly a general formula, it should reproduce any dependence observed in the coupling mechanism as a special case without introducing an additional factor.

Bargatze et al. (1986) analyzed the solar wind parameter dependence of the AL index, and showed that AL is expressed by $D^{1 / 6} V^{4 / 3} B \sin ^{4}(\theta / 2)$, whose exponents are consistent with the Vasyliunas et al. formula. Their $V^{4 / 3}$ dependence, however, is weaker than the results of Maezawa and Murayama (1986) and of Aoki (2005), who showed that the exponent for $V$ is close to two. Bargatze et al. further showed in their Fig. 4(b) that the $\sin ^{4}(\theta / 2)$ dependence is a good approximation. Close inspection of this figure, however, indicates that in the large values of $\theta$ (i.e., negative $B z$ ranges), $U(\theta) \cos \theta$, which corresponds to $B z$, agrees better with the data than $\sin ^{4}(\theta / 2)$. The $\sin ^{4}(\theta / 2)$ dependence agrees better with the data than $U(\theta) \cos \theta$ in the small $\theta$ ranges, where the physical meaning of AL is unclear (Allen and Kroehl 1975; Kamide and Akasofu, 1983).

Recently, Koskinen and Tanskanen (2002) thoroughly reviewed the basic ideas on $\varepsilon$, and pointed out some unclear physical foundations on them.

\section{Conclusions}

We have examined the IMF dependence of the ring current injection rate calculated from the $D s t$ index by comparing the influence of $B z$ with that of the combination of $\sin (\theta / 2)$ and $B$ (or $B_{T}$ ). Main results are as follows: (1) The exponent for $B z$ shows higher consistency than that for $\sin (\theta / 2)$. Higher consistency is seen in (a) smaller variability in the exponent for $B z$ for the change in the range of $B z$, and in (b) much larger variability in the exponent for $\sin (\theta / 2)$ for the change in the combination of parameters. (2) We never obtain $B^{2} \sin ^{4}(\theta / 2)$ or $B_{T}^{2} \sin ^{4}(\theta / 2)$, which is the IMF dependence expected from the $\varepsilon$ parameter. (3) The ring current injection rate shows a very low correlation with the Alfven Mach number. (4) The ring current injection rate has the $B y-\chi$ effect, which is an effect asymmetric with respect to the sign of $B y$. From the above results we conclude that the $\varepsilon$ parameter and the Vasyliunas et al. (1982) general formula are less appropriate than a function of $B z$, and that the IMF dependence of the energy coupling function is described better by $B z$ than by the combination of $\sin (\theta / 2)$ and $B$ (or $B_{T}$ ). The above results and conclusions are the same as those obtained by Aoki (2005) through the analysis of the AL index. On the basis of the above considerations, we suggest that the coupling function is approximated by

$$
P=f(B y, \chi) B z^{\alpha} V^{\beta} D^{\gamma},
$$

where $\alpha \sim 1, \beta \sim 2, \gamma \sim 0.4$, and $f(B y, \chi)$ is a function for expressing the effects of $B y$ and of $\chi$.

Acknowledgments. Data of the interplanetary magnetic field, the solar wind, and the Dst index were provided by Dr. J. H. King through the WDC-A for Rockets and Satellites in GSFC/NASA.

\section{Appendix.}

In this appendix, we supplement the discussion in Section 4.1 on the Vasyliunas et al. general formula and on the idea that the coupling function is described by the combination of $\left(B_{(T)}, \sin (\theta / 2), V, D\right)$ with more detailed analysis.

Table A1(a) shows correlation coefficients for $M_{A}$ and for the combination including $M_{A}$ for various ranges of $B z$ and for $V<600 \mathrm{~km} / \mathrm{s}$. When we compare the correlation coefficients with those for $\left(B z^{\prime}, V, D\right)$ in Table 2, we see that $\left(M_{A}\right),\left(M_{A}, \sin (\theta / 2)\right)$, and $\left(M_{A}, \sin (\theta / 2), V\right)$ have much lower correlations than $\left(B z^{\prime}, V, D\right)$. However, the combination $\left(M_{A}, \sin (\theta / 2), V, D\right)$ suddenly shows great improvement in correlation coefficients compared with $\left(M_{A}, \sin (\theta / 2), V\right)$, and the correlation coefficients of $\left(M_{A}, \sin (\theta / 2), V, D\right)$ are a little higher, by 0.07 at the maximum value, than those of $\left(B z^{\prime}, V, D\right)$. (The correlation coefficients of $\left(M_{A}^{\prime}, \sin (\theta / 2), V, D\right)$ are lower than $\left(B z^{\prime}, V, D\right)$, as seen in Table A1(b).)

Here we consider the reason for the higher correlation of $\left(M_{A}, \sin (\theta / 2), V, D\right)$ than $\left(B z^{\prime}, V, D\right)$. It is worth noting the following three points: First, in the regression analysis, the result of $\left(M_{A}, \sin (\theta / 2), V, D\right)$ is equivalent to that of $\left(B_{T}, \sin (\theta / 2), V, D\right)$ in the sense that for every range of $B z$, exponents for $\left(M_{A}, \sin (\theta / 2), V, D\right)$ can be derived from exponents for $\left(B_{T}, \sin (\theta / 2), V, D\right)$, and vice versa. This is easily confirmed by Tables A1(a) and $\mathrm{A} 1(\mathrm{~b})$ and simple calculations. Second, the assumption that $\left(M_{A}, \sin (\theta / 2), V, D\right)$ works in the solar windmagnetosphere coupling is not equivalent to the idea that $\left(B_{T}, \sin (\theta / 2), V, D\right)$ works. The latter includes the former, but the reverse is not true. Third, there is no reason that we should include $D$ in the regression analysis when we want to judge whether or not the combination $\left(M_{A}, \sin (\theta / 2)\right)$ works in the coupling. From the above three points, we cannot interpret that the high correlation of $\left(M_{A}, \sin (\theta / 2), V, D\right)$ represents the effectiveness of $\left(M_{A}, \sin (\theta / 2)\right)$ in the coupling, but should consider that $\left(B_{T}, \sin (\theta / 2), V, D\right)$ has a little better correlation than $\left(B z^{\prime}, V, D\right)$. The reasons for the higher correlation of $\left(B_{T}, \sin (\theta / 2), V, D\right)$ are probably considered as follows: The effect of $B y$ shown in Fig. 3 can be included in $B_{T}$ and improve the correlation. Larger number of parameters in the combination $\left(B_{T}, \sin (\theta / 2), V, D\right), 4$, than that of $\left(B z^{\prime}, V, D\right), 3$, might produce higher correlations. In any case, it should be pointed out again that the idea of describing the coupling by $\left(B_{T}, \sin (\theta / 2), V, D\right)$ has a difficulty in expressing the $B y-\chi$ effect, as mentioned in Section 4.1.

\section{References}

Allen, J. H. and H. W. Kroehl, Spatial and temporal distributions of mag- 
Table A.1. (a) Exponents and correlation coefficients (c.c.) for $M_{A},\left(M_{A}, \sin (\theta / 2)\right),\left(M_{A}, \sin (\theta / 2), V\right)$, and $\left(M_{A}, \sin (\theta / 2), V, D\right)$ for various ranges of $B z$ and for $V<600 \mathrm{~km} / \mathrm{s}$. (b) Same as (a) but for $\left(M_{A}^{\prime}, \sin (\theta / 2), V, D\right)$ and $\left(B_{T}, \sin (\theta / 2), V, D\right)$.

\begin{tabular}{|c|c|c|c|c|c|c|c|c|c|c|c|c|c|c|}
\hline \multirow{2}{*}{$\begin{array}{c}\text { (a) } \\
B z \text { range }\end{array}$} & \multicolumn{2}{|c|}{$M_{A}$} & \multicolumn{3}{|c|}{$\left(M_{A}, \sin (\theta / 2)\right)$} & \multicolumn{4}{|c|}{$\left(M_{A}, \sin (\theta / 2), V\right)$} & \multicolumn{5}{|c|}{$\left(M_{A}, \sin (\theta / 2), V, D\right)$} \\
\hline & $\alpha$ & c.c.* ${ }^{*}$ & $\alpha_{1}$ & $\alpha_{2}$ & c.c.* & $\alpha_{1}$ & $\alpha_{2}$ & $\alpha_{3}$ & c.c. $^{*}$ & $\alpha_{1}$ & $\alpha_{2}$ & $\alpha_{3}$ & $\alpha_{4}$ & c.c. ${ }^{*}$ \\
\hline$[-6, \quad 0]$ & -0.111 & -0.060 & -0.164 & 0.863 & 0.086 & -0.293 & 1.461 & 1.470 & 0.279 & -0.779 & 3.311 & 2.921 & 0.741 & 0.490 \\
\hline$[-7,0]$ & -0.163 & -0.088 & -0.236 & 1.341 & 0.131 & -0.359 & 1.909 & 1.503 & 0.299 & -0.810 & 3.484 & 2.951 & 0.756 & 0.516 \\
\hline$\left[\begin{array}{ll}, & 0\end{array}\right]$ & -0.342 & -0.185 & -0.434 & 2.535 & 0.260 & -0.539 & 3.011 & 1.619 & 0.381 & -0.864 & 3.765 & 2.969 & 0.774 & 0.591 \\
\hline$[-6,-1]$ & -0.112 & -0.060 & -0.168 & 0.881 & 0.087 & -0.290 & 1.430 & 1.484 & 0.281 & -0.786 & 3.328 & 2.919 & 0.743 & 0.491 \\
\hline$[-7,-1]$ & -0.165 & -0.088 & -0.240 & 1.366 & 0.132 & -0.358 & 1.884 & 1.514 & 0.301 & -0.817 & 3.501 & 2.949 & 0.758 & 0.517 \\
\hline$[\quad,-1]$ & -0.345 & -0.185 & -0.440 & 2.574 & 0.262 & -0.540 & 3.002 & 1.625 & 0.382 & -0.870 & 3.780 & 2.962 & 0.776 & 0.592 \\
\hline$[-6,-2]$ & -0.012 & -0.006 & -0.008 & -0.049 & 0.006 & -0.103 & 0.239 & 1.570 & 0.297 & -0.959 & 3.983 & 3.019 & 0.823 & 0.497 \\
\hline$[-7,-2]$ & -0.073 & -0.036 & -0.115 & 0.652 & 0.054 & -0.205 & 0.917 & 1.594 & 0.303 & -0.985 & 4.144 & 3.035 & 0.835 & 0.524 \\
\hline$[\quad,-2]$ & -0.309 & -0.153 & -0.414 & 2.471 & 0.217 & -0.481 & 2.625 & 1.697 & 0.372 & -1.004 & 4.291 & 2.977 & 0.831 & 0.607 \\
\hline
\end{tabular}

*correlation coefficient.

\begin{tabular}{|c|c|c|c|c|c|c|c|c|c|c|}
\hline \multirow{2}{*}{$\begin{array}{c}\text { (b) } \\
B z \text { range }\end{array}$} & \multicolumn{5}{|c|}{$\left(M_{A}^{\prime}, \sin (\theta / 2), V, D\right)$} & \multicolumn{5}{|c|}{$\left(B_{T}, \sin (\theta / 2), V, D\right)$} \\
\hline & $\alpha_{1}$ & $\alpha_{2}$ & $\alpha_{3}$ & $\alpha_{4}$ & c.c. ${ }^{*}$ & $\alpha_{1}$ & $\alpha_{2}$ & $\alpha_{3}$ & $\alpha_{4}$ & c.c. ${ }^{*}$ \\
\hline$\left[\begin{array}{ll}-6, & 0\end{array}\right]$ & -0.842 & 2.280 & 2.536 & 0.740 & 0.458 & 0.779 & 3.311 & 2.142 & 0.351 & 0.490 \\
\hline$\left[\begin{array}{ll}-7, & 0\end{array}\right]$ & -0.905 & 2.559 & 2.583 & 0.770 & 0.482 & 0.810 & 3.484 & 2.141 & 0.351 & 0.516 \\
\hline$\left[\begin{array}{ll}, & 0\end{array}\right]$ & -1.043 & 3.060 & 2.665 & 0.830 & 0.559 & 0.864 & 3.765 & 2.105 & 0.342 & 0.591 \\
\hline$[-6,-1]$ & -0.844 & 2.282 & 2.544 & 0.740 & 0.459 & 0.180 & 3.328 & 2.133 & 0.350 & 0.491 \\
\hline$[-7,-1]$ & -0.907 & 2.564 & 2.590 & 0.771 & & & 3.501 & & 0.350 & 0.517 \\
\hline$[\quad,-1]$ & -1.046 & 3.071 & 2.668 & 0.831 & 0.560 & 0.870 & 3.780 & 2.092 & 0.341 & 0.592 \\
\hline$[-6,-2]$ & -0.820 & 2.286 & 2.575 & 0.722 & 0.464 & 0.959 & 3.983 & 2.060 & 0.344 & 0.497 \\
\hline$[-7,-2]$ & -0.905 & 2.698 & 2.627 & 0.762 & 0.488 & 0.985 & 4.144 & 2.050 & 0.343 & 0.524 \\
\hline$[\quad,-2]$ & -1.079 & 3.428 & 2.694 & 0.836 & 0.574 & 1.004 & 4.291 & 1.973 & 0.329 & 0.607 \\
\hline
\end{tabular}

*correlation coefficient.

netic effects of auroral electrojets as derived from AE indices, J. Geophys. Res., 80, 3667-3677, 1975.

Aoki, T., Influence of the dipole tilt angle on the development of auroral electrojets, J. Geomag. Geoelectr., 29, 441-453, 1977.

Aoki, T., On the validity of Akasofu's $\varepsilon$ parameter and of the Vasyliunas et al. general formula for the rate of solar wind-magnetosphere energy input, Earth Planets Space, 57, 131-137, 2005.

Baker, D. N., R. D. Zwickl, S. J. Bame, E. W. Hones, Jr., B. T. Tsurutani, E. J. Smith, and S.-I. Akasofu, An ISEE 3 high time resolution study of interplanetary parameter correlations with magnetospheric activity, J. Geophys. Res., 88, 6230-6242, 1983.

Bargatze, L. F., R. L. McPherron, and D. N. Baker, Solar windmagnetosphere energy input functions, in Solar Wind-Magnetosphere Coupling, edited by Y. Kamide and J. A. Slavin, pp. 101-109, Terrapub/Reidel, Tokyo, 1986.

Burton, R. K., R. L. McPherron, and C. T. Russell, An empirical relationship between interplanetary conditions and Dst, J. Geophys. Res., 80, 4204-4214, 1975.

Dessler, A. J. and E. N. Parker, Hydromagnetic theory of geomagnetic storms, J. Geophys. Res., 64, 2239-2252, 1959.

Fairfield, D. H. and L. J. Cahill, Jr., Transition region magnetic field and polar magnetic disturbances, J. Geophys. Res., 71, 155-169, 1966.

Feldstein, Y. I., V. Yu. Pisarsky, N. M. Rudneva, and A. Grafe, Ring current simulation in connection with interplanetary space conditions, Planet. Space Sci., 32, 975-984, 1984.

Fenrich, F. R. and J. G. Luhmann, Geomagnetic response to magnetic clouds of different polarity, Geophys. Res. Lett., 25, 2999-3002, 1998.

Gonzalez, W. D., B. T. Tsurutani, A. L. C. Gonzalez, E. J. Smith, F. Tang, and S.-I. Akasofu, Solar wind-magnetosphere coupling during intense magnetic storms (1978-1979), J. Geophys. Res., 94, 8835-8851, 1989.

Gonzalez, W. D., J. A. Joselyn, Y. Kamide, H. W. Kroehl, G. Rostoker, B.
T. Tsurutani, and V. M. Vasyliunas, What is a geomagnetic storm?, $J$. Geophys. Res., 99, 5771-5792, 1994.

Kamide, Y. and S.-I. Akasofu, Notes on the auroral electrojet indices, Rev. Geophys. Space Phys., 21, 1647-1656, 1983.

Koskinen, H. E. J. and E. I. Tanskanen, Magnetospheric energy budget and the epsilon parameter, J. Geophys. Res., 107(A11), 1415, doi:10.1029/ 2002JA009283, 2002.

Maezawa, K., Statistical study of the dependence of geomagnetic activity on solar wind parameters, in Quantitative Modeling of Magnetospheric Processes, Geophys. Monogr. Ser., vol. 21, edited by W. P. O1son, pp. 436-447, AGU, Washington, D. C., 1979.

Maezawa, K. and T. Murayama, Solar wind velocity effects on the auroral zone magnetic disturbances, in Solar Wind-Magnetosphere Coupling, edited by Y. Kamide and J. A. Slavin, pp. 59-83, Terrapub/Reidel, Tokyo, 1986.

Murayama, T., Coupling function between the solar wind and the Dst index, in Solar Wind-Magnetosphere Coupling, edited by Y. Kamide and J. A. Slavin, pp. 119-126, Terrapub/Reidel, Tokyo, 1986.

Murayama, T and K. Hakamada, Effects of solar wind parameters on the development of magnetospheric substorms, Planet. Space Sci., 23, 7591, 1975.

Murayama, T., T. Aoki, H. Nakai, and K. Hakamada, Empirical formula to relate the auroral electrojet intensity with interplanetary parameters, Planet. Space Sci., 28, 803-813, 1980.

Nakai, H., Influence of the transverse component of the interplanetary magnetic field on the size of the auroral oval, J. Geomag. Geoelectr., 39, 501-519, 1987.

Neugebauer, M. and C. W. Synder, Mariner 2 observations of the solar wind, 1, Average properties, J. Geophys. Res., 71, 4469-4484, 1966.

O'Brien, T. P. and R. L. McPherron, An empirical phase space analysis of ring current dynamics: Solar wind control of injection and decay, $J$. 
Geophys. Res., 105, 7707-7719, 2000.

O'Brien, T. P. and R. L. McPherron, Seasonal and diurnal variation of Dst dynamics, J. Geophys. Res., 107(A11), 1341, doi:10.1029/ 2002JA009435, 2002.

Perreault, P. and S.-I. Akasofu, A study of geomagnetic storms, Geophys. J. R. Astron. Soc., 54, 547-573, 1978.

Rostoker, G., H.-L. Lam, and W. D. Hume, Response time of the magnetosphere to the interplanetary electric field, Can. J. Phys., 50, 544-547, 1972.

Sckopke, N., A general relation between the energy of trapped particles and the disturbance field near the Earth, J. Geophys. Res., 71, 31253130, 1966.

Temerin, M. and X. Li, A new model for the prediction of Dst on the basis of the solar wind, J. Geophys. Res., 107(A12), 1472, doi:10.1029/ 2001JA007532, 2002.

Vasyliunas, V. M., J. R. Kan, G. L. Siscoe, and S.-I. Akasofu, Scaling relations governing magnetospheric energy transfer, Planet. Space Sci., 30, 359-365, 1982.

Wu, J.-G. and H. Lundstedt, Geomagnetic storm predictions from solar wind data with the use of dynamic neural networks, J. Geophys. Res., 102, 14255-14268, 1997a.

Wu, J.-G. and H. Lundstedt, Neural network modeling of solar windmagnetosphere interaction, J. Geophys. Res., 102, 14457-14466, 1997 b.

T. Aoki (e-mail: aoki317@ruby.ocn.ne.jp) 